\title{
Genetics behind crop mimicry
}

Nat. Ecol. Evol. 3, 1474-1482 (2019).

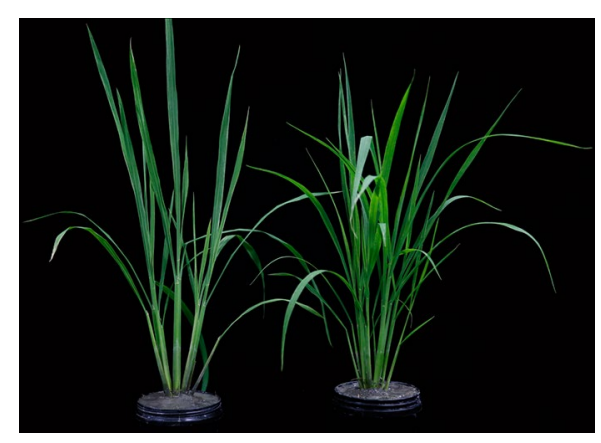

Credit: Image courtesy of Dongya Wu

Echinochloa weeds generally display loose or prostrate plant architectures in the wild. In paddy fields, however, they often evolve phenotypes, such as a small tiller angle, to resemble the seedlings of cultivated rice - a phenomenon termed 'Vavilovian mimicry'. Vavilovian mimicry has been hypothesized to be driven by unintentional human selection, but its evolutionary mechanism remains unexplored. By analysing the genomes of 328 Echinochloa crus-galli accessions collected from diverse locations on the Yangtze River, Longjiang Fan's group, from Zhejiang University, China, and collaborators now obtain novel insights into the evolution of this interesting mimicry.

To assess the mimicry level of these weed accessions, the researchers developed a mimicry index (MI) by calculating the weighted score for four typical mimicry traits (small tiller angle, straight stem node, green stem base and compact leaves). An MI score was then assigned to each accession based on phenotypic data collected in two seasons.

The genomes of these lines were re-sequenced and single nucleotide polymorphic sites as well as insertions and deletions were identified. Based on these variations, the 328 accessions were classified into three major groups: mimetic, nonmimetic and mixed groups. The mimetic and non-mimetic groups have a high and low MI value, respectively. According to phylogenetic and diversity analyses, the mimetic group was derived from the nonmimetic group and has experienced genetic bottleneck. The divergence time between the two groups was estimated to be about 1,000 years ago.

Regions with positive selection signatures were identified, containing 1,986 candidateselected genes. Among these, 87 genes were related to plant architecture, including a key gene, $L A Z Y 1$, known to control tiller angle. The results provided the first genomic evidence for Vovilovian mimicry.

This study advances our understanding of mimicry adaptation and the origin of agricultural weeds.

\section{Jun Lyu}

Published online: 11 November 2019 https://doi.org/10.1038/s41477-019-0557-y 